\title{
CONTROL OF POWER SYSTEMS WITH FACTS DEVICES CONSIDERING DIFFERENT LOAD CHARACTERISTICS
}

\author{
Ingo Winzenick*, Michael Fette**, Joachim Horn* \\ *Helmut-Schmidt-University / University of the Federal Armed Forces Hamburg \\ Department of Electrical Engineering, Institute of Automatic Control Engineering \\ Holstenhofweg 85, D-22043 Hamburg, Germany \\ ** System \& Dynamik Beratungsunternehmen \\ Tempelhoferstraße 37, D-33100 Paderborn, Germany
}

\begin{abstract}
Power systems form one of the largest and most complex technical systems. Changes of load parameters may lead to so called bifurcations, resulting in nonlinear phenomena like voltage collapse or interarea oscillations. Special software tools are able to simulate these systems and to identify bifurcations. This paper presents a systematic approach to determine gain factors of Flexible AC Transmission Devices (FACTS) considering changes of load parameters. This approach is applied to two different power system models.
\end{abstract}

Copyright @ IFAC 2005

Keywords: Nonlinear Systems, Power Systems, Load Model, Voltage Collapse, Critical Points, Hopf Bifurcation

\section{INTRODUCTION}

Nonlinear phenomena in power systems like voltage collapse or interarea oscillations have been investigated by many researchers (Ajjarapu et al, 1992; Budd et al, 2002; Chiang et al, 1990; Chiang et al, 1993; Dobson et al, 1989; Seydel, 2001). Understanding these phenomena is crucial because they can cause critical situations up to blackouts. The years 2003 and 2004 showed in a very dramatical way that reliable electric power is essential especially for developed nations (VDE, 2003).

In order to prevent such situations many efforts are done to analyze and control the system. Because of various trends and effects this is more complicated than ever before. Ecological reasons lead to an increasing implementation of renewable energy power stations. The dynamic behaviour of wind farms, photoelectric cells, natural gas stations, etc. differ qualitatively from conventional power stations. Some of them even introduce complicated stochastic processes. Economical reasons force the power industry to reduce their overcapacity in order to increase their competitiveness. As a result, the systems operate more at their physical limits than ever before. Due to this and the increasing demand of electric energy, nonlinear effects become more and more dominant.

One possibility to face these problems is to use sophisticated analysis tools and simulation methods. So called continuation methods are very helpful to investigate the system and its dynamical behaviour qualitatively. Continuation methods and a special bifurcation are discussed in section 2 . 
Section 3 presents two examples of power system models. These models were used by many researchers, the authors used it as an example system in a former paper (Fette et al). This paper discuss a new approach to consider load parameter variations and thus different load characteristics.

Chapter 4 explains the approach and illustrates it by using at first a three node system. The nine node system is considered additionally in order to illustrate the applicability on more complex systems. A summary close this paper.

\section{CONTINUATION METHODS AND BIFURCATIONS}

Continuation Methods: Even in a simple representation of an electric power system (the load flow equations) classical calculation methods based on NewtonRaphson-principles cannot ensure that all numeric solutions are found. These methods are of fractal nature (cp. Thorp et al, 1989; Thorp et al, 1990).

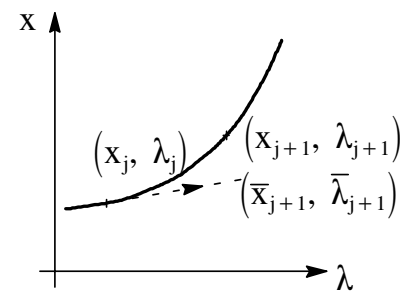

Fig. 1 Predictor-Corrector-Principle

Continuation methods solve this problem. They become more and more usable even in electric power system studies because of the still increasing computational power. Additionally they are capable to analyze systems if parameters (one is interested in) are varied.

Starting from an initial solution which can be calculated e.g. by using classical Newton based algorithms a first prediction is calculated. Subsequent additional correction steps have to be done (see Fig. 1). The interested reader is referred to e.g. (Kuznetsov, 1995; Seydel, 1994). With special test functions a classification of each calculated point as well as of its corresponding dynamical behaviour is possible.

Bifurcations: The so called Hopf bifurcation is known as a possible cause of nonlinear oscillation phenomena and -due to this- is of special interest.

Beneath a critical parameter value a stable solution exists, see Fig. 2. This solution bifurcates at the critical point (the bifurcation point) into an unstable solution, and a stable limit cycle occurs. This behavior is caused by a pair of complex eigenvalues which crosses the imaginary axis when a parameter $(\lambda)$ changes a critical value (Seydel, 1994).

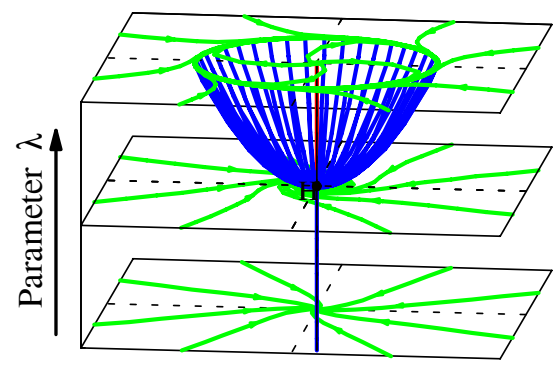

Fig. 2 Hopf Bifurcation

\section{POWER SYSTEM MODELS AND FACTS DEVICES}

Three Node System: This subsection presents a widely used example of a three node power system. Although quite simple, it shows an interesting behaviour and can be used to present the main ideas and concepts.

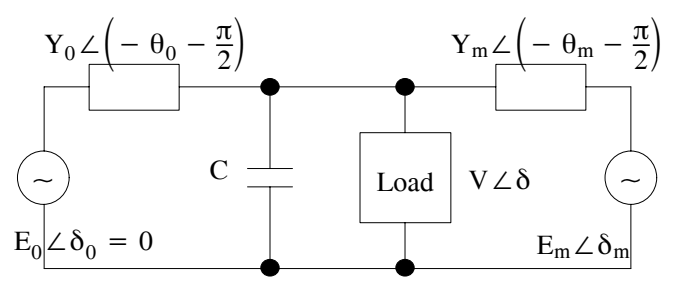

Fig. 3 Three Node Model

The three node model consists of two generators and a load. One generator represents an infinite bus, the other one an arbitrary machine. Together they feed the dynamical load. This model was used in various studies and its differential equations are given by

$$
\begin{aligned}
\dot{\delta}_{m}= & \omega, \\
M \dot{\omega}= & -d_{m} \omega+P_{m}+E_{m}^{2} Y_{m} \sin \left(\theta_{m}\right) \\
& +E_{m} Y_{m} V \sin \left(\delta-\delta_{m}-\theta_{m}\right), \\
K_{q \omega} \dot{\delta}= & Q_{d}-Q_{0}-Q_{1}-K_{q v} V-K_{q v 2} V^{2}, \\
K_{p v} T \dot{V}= & P_{d}-P_{0}-P_{1}-K_{p v} V-\frac{K_{p \omega}}{K_{q \omega}}\left(Q_{d}\right. \\
& \left.-Q_{0}-Q_{1}-K_{q v} V-K_{q v 2} V^{2}\right),
\end{aligned}
$$

with the algebraic constraints

$$
\begin{aligned}
P_{d}= & -E_{o}^{\prime} Y_{0}^{\prime} V \sin \left(\delta+\theta_{0}^{\prime}\right) \\
& -E_{m} Y_{m} V \sin \left(\delta-\delta_{m}+\theta_{m}\right) \\
& +\left(Y_{0}^{\prime} \sin \left(\theta_{0}^{\prime}\right)+Y_{m} \sin \left(\theta_{m}\right)\right) V^{2}, \\
Q_{d}= & E_{o}^{\prime} Y_{0}^{\prime} V \cos \left(\delta+\theta_{0}^{\prime}\right) \\
& +E_{m} Y_{m} V \cos \left(\delta-\delta_{m}+\theta_{m}\right) \\
& -\left(Y_{0}^{\prime} \cos \left(\theta_{0}^{\prime}\right)+Y_{m} \cos \left(\theta_{m}\right)\right) V^{2}
\end{aligned}
$$

$M, d_{m}$ and $P_{m}$ are the generator inertia constant, damping coefficient, and mechanical power, respectively. $K_{i}$ and $T$ denote the load parameters. The voltages, admittances and angles of the network and/or generators are represented by $V, E_{i}, Y_{i}$ and $\delta_{i}$.

The results presented in this paper are calculated using the same parameter set as in (Dobson et al, 1989). 


$$
\begin{gathered}
P_{d}=P_{0}+P_{1}+K_{p \omega} \dot{\delta}+K_{p V}(V+T \dot{V}) \\
Q_{d}=Q_{0}+Q_{1}+K_{q \omega} \dot{\delta}+K_{q V} V+K_{q V 2} V^{2}
\end{gathered}
$$

In order to complete the description the load model equations are shown in (4).

Nine Node System: Another example of a power system model is shown in Fig. 4. It is presented in detail in (Anderson et al, 1977). Additional to it a dynamic load model is installed at the marked node.

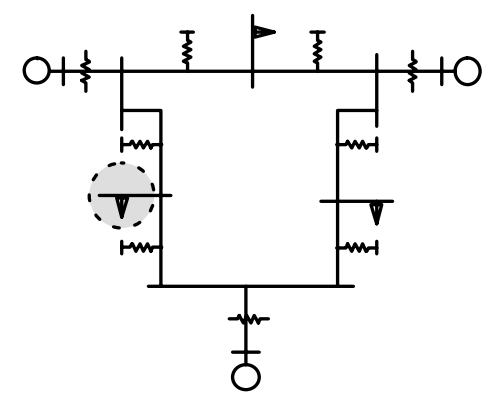

Fig. 4 Nine Node System (Structure)

The equations of the dynamic load model as well as the corresponding used parameter sets are based on (Dobson et al, 1992).

$$
\begin{gathered}
P_{d}=l c p P F+d \dot{\delta}+a \cdot \dot{V} \\
Q_{d}=l c p \sqrt{1-P F^{2}}+b \dot{\delta}+k \cdot \dot{V}+Q_{1}
\end{gathered}
$$

In principle the equations (5) and (6) represents the same structure of the load as used within the three node network.

Flexible AC Transmission Systems (FACTS): In order to change the location of bifurcation points possibilities to influence the system behaviour are needed. Special devices called FACTS are capable to change parameters of the power system like node voltages, phase angles or impedances.

In principle FACTS are power electronic devices which are capable to store energy. This energy is used for controlling tasks within a short time period. A lot of different types of FACTS are known. Some of them are used since several years, the other ones are still under development (Hingorani et al, 2000). Three devices -the Static VAr Compensator (SVC), the Phase Angle Regulator (PAR) and the Controlled Series Compensator (CSC)- are considered.

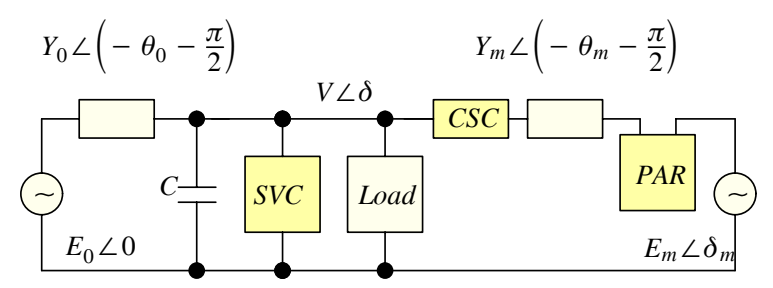

Fig. 5 Three Node System with installed FACTS
They correspond in a special way to the needs of bifurcation control. Each device affects only one parameter. Additionally they are fully developed and many experiences was made in other applications like compensation and damping tasks for several years. Thus these devices are well known in contrast to devices like the UPFC. All considered devices are modelled as an first order system (7) where the output signal $Y$ is limited additionally (Srivatstava et al, 1998)

$$
T \dot{Y}=K_{s}\left(U_{r}-U_{m}\right)-Y .
$$

As an error signal the acceleration power of the generator is used respectively the deviation of the frequency. $U_{r}$ represents the reference value and $U_{m}$ the measured one. $T$ denotes the time constant of the device and $K_{S}$ denotes its gain. The installation of the chosen devices into the three node system is shown in Fig. 5. Due to page limitations, only the results for the Static VAr Compensator (SVC) are presented.

\section{ANALYSIS STRATEGY AND RESULTS}

Detailed information about the system and its dynamic behaviour is always needed before any device or strategy can be changed or installed. The dynamic behaviour is represented here by bifurcations. As noted before Hopf bifurcations are known as possible critical causes of oscillation phenomena and should be avoided. In order to do this, the following steps are proposed:

a) Determine a first equilibrium solution

b) Calculate an equilibrium curve in dependency of a parameter of interest

c) Detect critical and uncritical bifurcations

d) Choose appropriate devices which are capable to control the critical bifurcations

e) Calculate further bifurcation curves at different values of a parameter of interest

f) Determinate gain limits based on the former calculated curves

g) Identification and determination of possible operation areas

h) Define stability margins because of safety aspects

Application of this strategy is illustrated first by using the three node power system model.

\subsection{Control of the Three Node System}

The required first solution (step a) was calculated by using a classical Newton based simulation method (Fette, 2001). Starting from this point the following calculation uses the reactive power demand of the load 
$\mathrm{Q}_{1}$ as a continuation parameter (step b) . The result is shown in Fig. 6.

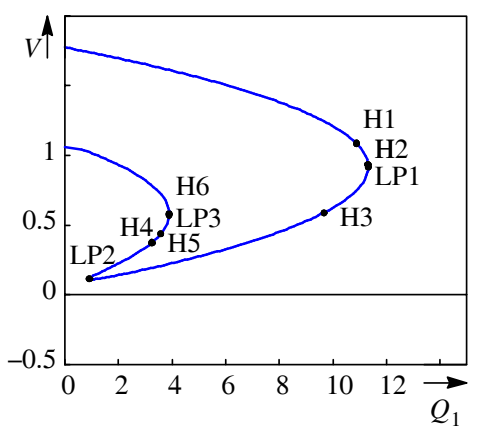

Fig. 6 Three Node System: Bifurcation Diagram

The upper branch up to the Saddle Node or Limit Point bifurcation (LP1) represents the normal operation area of the system. Within this area Hopf bifurcations are detected (step c). One can see that the stable area of operation is bounded by a first Hopf bifurcation (H1) and limited absolutely by the saddle node bifurcation (LP1). Former studies (Srivastava et al, 1998) showed that it is in principle possible to influence these bifurcations via active elements like FACTS devices.

Three Node Network with FACTS device: As noted before the SVC is chosen (step d) because it is known that this device is capable to influence the Hopf bifurcation of interest. The accelerating power of one generator is chosen as the input signal and its gain is used as another bifurcation parameter.

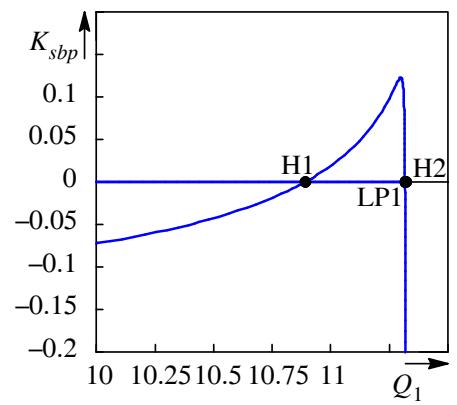

Fig. 7 Usage of FACTS

The result is shown in Fig. 7, where the horizontal line at zero gain represents the same bifurcation curve as shown in Fig. 6. Therefore H1, H2, and LP1 label the same bifurcations as shown before in Fig. 6. The second curve crossing $\mathrm{H} 1$ and $\mathrm{H} 2$ represents the continuation of these Hopf points in consideration of the FACTS gain $\mathrm{K}_{\text {sbp }}$ (step e). Obviously the Hopf bifurcation $\mathrm{H} 1$ and $\mathrm{H} 2$ change their locations and at a value of about 0.12 for the gain of the FACTS device they collide and disappear (step f) .

Now the load characteristics are changed by varying a load parameter. The question is whether it is possible to prevent the Hopf bifurcations within an interval of the load parameter. The chosen load parameter is varied and the corresponding bifurcation diagrams (continued Hopf curves only) are printed (Fig. 8).

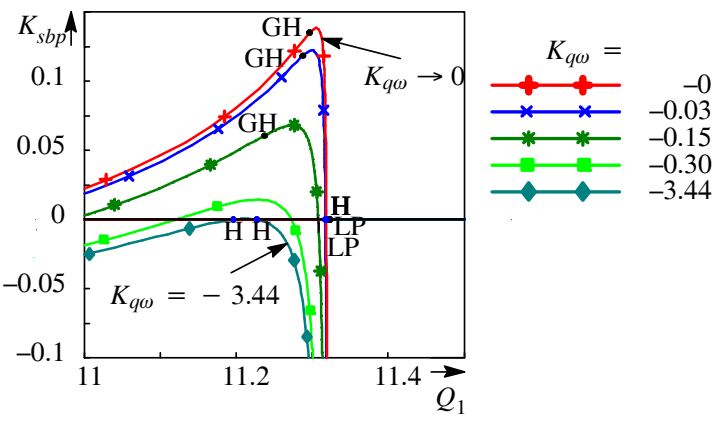

Fig. 8 Hopf Curves at Different Load Parameter

As a result, a parameter value $\left(\mathrm{K}_{\mathrm{qw}}<-3.44\right)$ exists where Hopf bifurcations do not appear. Within the interval $-3.44<\mathrm{K}_{\mathrm{qw}}<0$ it is possible to determine a gain value $\left(\mathrm{K}_{\mathrm{sbp}}=0.136\right)$ of the FACTS device which ensures that these bifurcations do not occur (step $g$ ) . As a matter of course the identified gain has to be adapted to the real FACTS device because of the usually used models of higher complexity.

Results: These results can be illustrated by using time series calculations as well. Fig. 9 shows the uncontrolled and controlled case. Both calculations are started at the same equilibrium of Fig. 6 between bifurcation $\mathrm{H} 1$ and $\mathrm{H} 2$. It is disturbed by a slight change of the voltage. Obviously, the considered equilibrium is unstable and results in a oscillation phenomenon without FACTS devices.

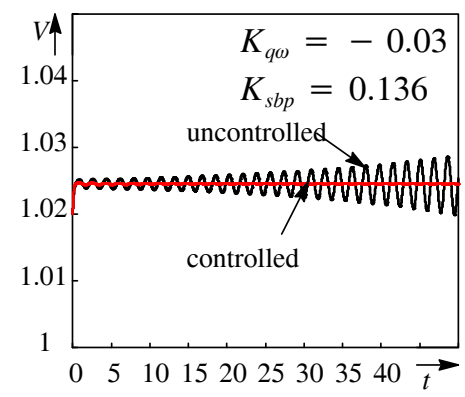

Fig. 9 System with and without FACTS-Device

By using the SVC device -designed in the presented way- the "controlled" graph is calculated. This case shows a stable solution. This is obtained even if the load parameter varies within the interval noted before.

\subsection{Control of the Nine Node System}

This strategy is now adopted to the second model of a power system. The first bifurcation diagram is calculated as before (Fig. 11). Again a load demand parameter (lcp) is chosen as a bifurcation parameter. Two Hopf bifurcations occur as well as a Limit Point. These points restrict the operation area.

Nine Node System with SVC: The first Hopf bifurcation (H1) is continued by using a gain of the installed SVC-device as another bifurcation parameter. Again it is possible to influence bifurcation $\mathrm{H} 1$ and $\mathrm{H} 2$ up to a gain value where they collide and disappear. This is 


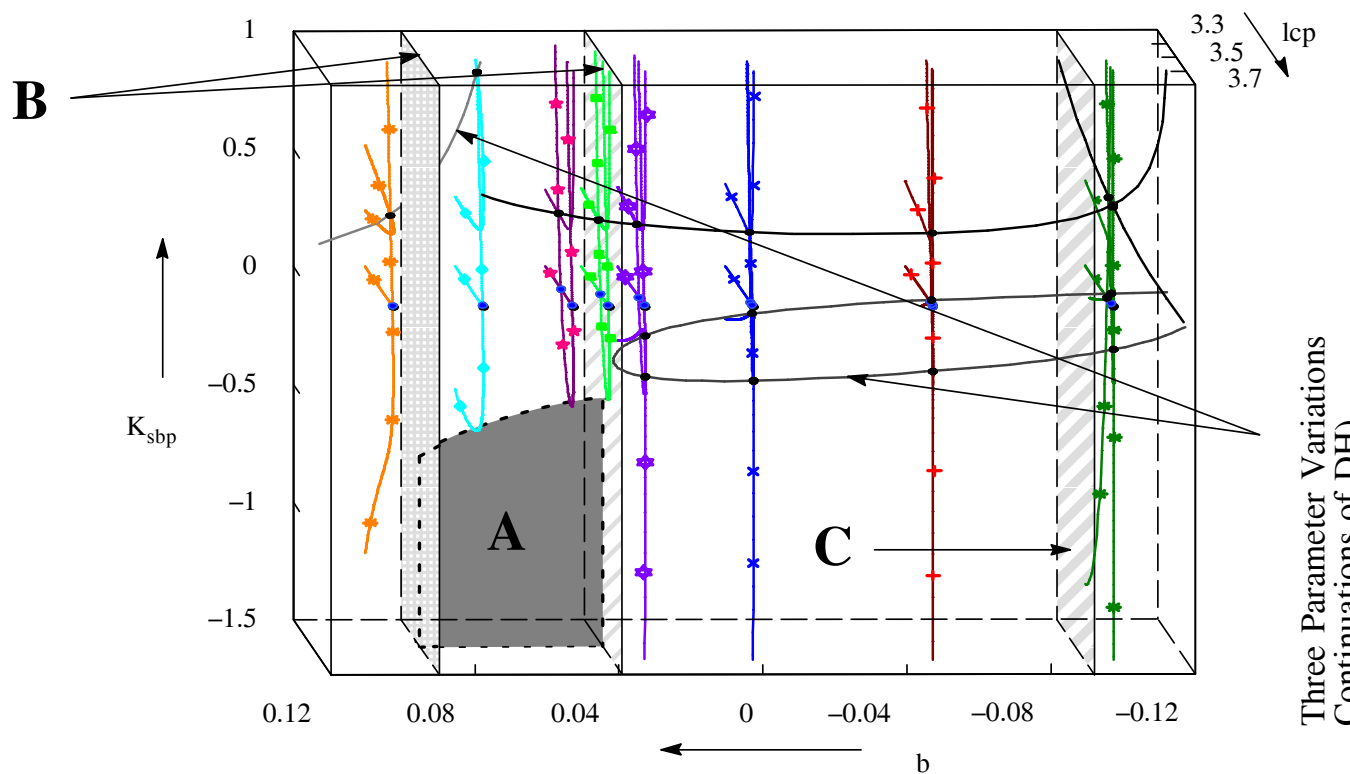

Fig. 10 Nine Node System: Three-Dimensional Bifurcation Diagram

shown in Fig. 12. Contrary to the three node model additional characteristic points occur (DH). These points represent Double Hopf (DH) bifurcations. That means that two Hopf-curves intersects. Further Hopf curves are displayed in the same figure.

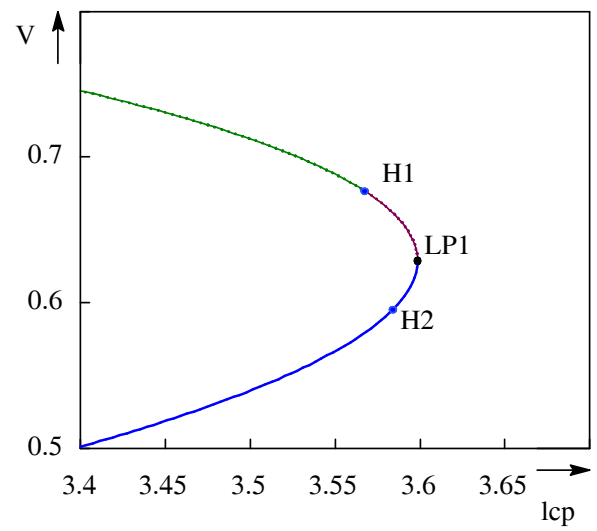

Fig. 11 Nine Node System: Bifurcation Diagram

Observation of these "new" curves shows that it is possible to increase the considered domain of operation of the system, but in this case it is not possible to expand it up to the absolute maximum at the limit point. The best case is marked by the dotted line in Fig. 12.

Results: As before load parameters are changed and solutions are identified where the stable domain of operation area is increased even if the load parameter changes. Because of the more complicated structure of the system a three dimensional diagram (Fig. 10) is plotted for a better visualization. It consists of multiple bifurcation diagrams like Fig. 12 at different values of the load parameter (b) which corresponds with the chosen parameter $\left(\mathrm{K}_{\mathrm{qw}}\right)$ before.

Again a parameter area and thus a FACTS gain can be determined where bifurcations are prevented completely and the area of operation can be increased (A). This is reached by analyzing the continued Hopf points as well as the usage of a continuation via third parameter of the double Hopf bifurcations.

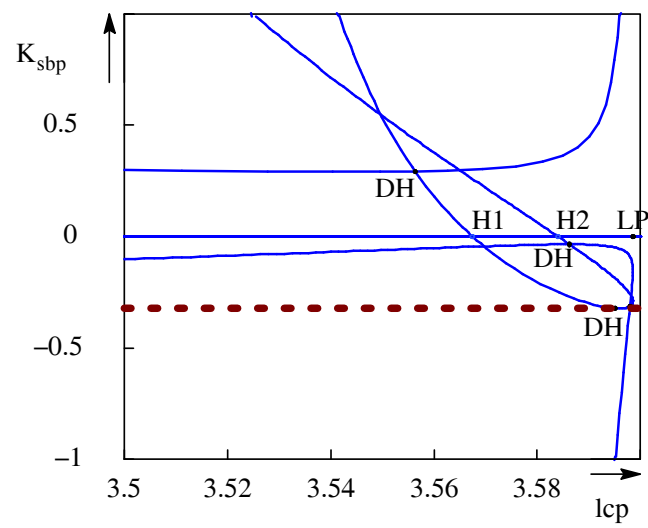

Fig. 12 Nine Node System:

Dependency of Load Parameter

Therewith it is possible to determine limits shown by $\mathrm{K}_{\mathrm{sbp}} / \mathrm{lcp}$ planes (B) and (C). Obviously it is possible to increase the domain of operation although a Hopf bifurcation remains up to the limit (C).

\subsection{Consequences}

The two examples show that continuation methods together with bifurcation analysis can be used to identify characteristic dynamic properties of the considered systems. By using an appropriate FACTS device it is possible to control bifurcations and based on further analysis domains of operation of these devices can be identified.

Because of safety reasons the margins of the identified operation areas are not useful in practice. Former simulations showed that situations can occur where 
oscillations emerge before the limit is reached. Because of this margins should be considered (step h) :

Definition: Bifurcation Margin

A Bifurcation Margin is a distance to the determined limit value or limit plane represented by critical bifurcations.

Because of former experiences a bifurcation margin of five percent is proposed. This means in the case of the nine node system that the grey shaded area (A) has to be reduced to get the real operation area at each limit margin.

Now one is capable to determine intervals of controller gains which ensure that no critical bifurcations occur respectively critical bifurcations are moved to higher values of the considered parameter and thus the area of operation is increased.

\section{SUMMARY}

Power systems are large complicated systems and nonlinear effects become more and more dominant. This paper presented a strategy to design FACTS devices in a structured way even if the loads vary in order to prevent critical bifurcations.

The used simulation method was briefly explained and the Hopf bifurcation known as a possible cause of nonlinear oscillation phenomena was described. The strategy which helps to determine the gain of FACTS devices by using bifurcation diagrams was presented. These FACTS devices influence system parameters and thus control bifurcations up to their disappearance.

Two examples of power system models were considered. One model represents a three node system and was used to illustrate the main ideas and principles. Next the strategy was adopted to a more complicated nine node system. Only the results for one FACTS device were presented, but the strategy can be adopted in principle to other devices too.

Critical regions of the system as well as critical areas of the controller gain can be identified by interpreting additional bifurcation diagrams. Therefore it is possible to identify a parameter region where one can ensure that the operation area is increased even if e.g. load parameters change.

Finally a definition of bifurcation margins was given which ensures the practical applicability of the presented strategy.

Additional to the identified and increased domain of operation it must be ensured that FACTS devices used in the presented way do not operate out of this domain. Because of new or moved bifurcations unwanted dynamic behaviour can occur and result in the worst case into collapse situations. If one wants to operate within this area again the bifurcation behaviour has to be analyzed.

\section{REFERENCES}

Ajjarapu, V., Lee, B. (1992). Bifurcation Theory and its Application to Nonlinear Dynamical Phenomena in an Electrical Power System. In: IEEE ToPS Vol. 7, No. 1, pp. 424-431

Anderson P. M.; Fouad A. A. (1977). Power System Control and Stability. 1st Ed IOWA, USA..

Budd, C. J., Wilson, J. P. (2002). Bogdanov-Takens Bifurcation Points and Sil'nikov Homoclinicity in a Simple Power System Model of Voltage Collapse. In: IEEE ToC\&S I, Vol. 49, No. 5, pp. 575-590

Chiang, H. D., Dobson, Ian, Thomas, R. J., Thorp, J. S., Fekih-Ahmed, Lazhar (1990). On Voltage Collapse in Electric Power Systems. In: IEEE ToPS, Vol. 5, No. 2, pp. 601-611

Chiang, H. D., Liu, C. W., Varaiya, P. P., Wu, F.F., Lauby, M. G. (1993). Chaos in a Simple Power System. In: IEEE ToPS, Vol. 8, pp. 1407-1417

Dobson, I., Alvarado, F. DeMarco, C. L. (1992). Sensitivity of Hopf Bifurcations to Power System Parameters. In: Proc. of the 31st Conference on Decision and Control, pp. 2928-2933

Dobson, I., Chiang, H. D. (1989). Towards a Theory of Voltage Collapse in Electric Power Systems. In: Systems \& Control Letters, Vol. 13, pp. 253-262

Fette, M. (2001). Dynamik nichtlinearer Elektroenergiesysteme. Habilitationsschrift, Universität Paderborn, Fachbereich Elektrotechnik

Fette, M., Winzenick, I. (to be published). Bifurcation Analysis of Power System Load Characteristics with Continuation Methods. In: MCMDS

Hingorani, N. G., Gyugyi, L. (2000). Understanding FACTS. IEEE Press

IEEE Task Force on Load Representation for Dynamic Performance (1993). Load Representation for Dynamic Performance Analysis. In: IEEE ToPS, Vol. 8, No. 2, pp. 472-482

Kuznetsov, Y. A. (1995). Elements of Applied Bifurcation Theory. Springer New York

Seydel, R. (1994). Practical Bifurcation and Stability Analysis. Springer New York

Seydel, R. (2001). Assessing Voltage Collapse. In: Special Issue on Bifurcation Control: Methodologies and Applications. 31, 3, pp. 171-176

Srivastava, K. N. Srivastava, S. C. (1998). Elimination of Dynamic Bifurcation and Chaos in Power Systems Using Facts Devices. In: IEEE ToC\&S I, Vol. 45, No. 1, pp. 72-78

Thorp, J. S., Naqavi, S.A. (1989). Load Flow Fractals. In: Proc. of the 28th Conference on Decision and Control, pp. 1822-1827

Thorp, J. S., Naqavi, S. A., Chiang, H. D. (1990). More Load Flow Fractals. In: Proc. of the 29th Conference on Decision and Control, pp. 3028-3030

VDE-Analyse (2003). Stromversorgungsstörungen 